\title{
Experimental validation of image contrast correlation between ultra-small-angle $X$-ray scattering and grating-based dark-field imaging using a laser-driven compact $X$-ray source
}

\author{
Experimentelle Verifizierung des Zusammenhangs zwischen \\ Röntgen-Kleinwinkelstreuung und gitter-basierter \\ Röntgen-Dunkelfeldbildgebung unter Verwendung eines \\ laser-getriebenen Kompaktsynchrotrons
}

\author{
Martin Bech ${ }^{1, *}$, Simone Schleede ${ }^{1}$, Guillaume \\ Potdevin', Klaus Achterhold ${ }^{1}$, Oliver Bunk², \\ Torben H. Jensen ${ }^{3}$, Rod Loewen ${ }^{4}$, Ron Ruth ${ }^{4,5}$ \\ and Franz Pfeiffer ${ }^{1}$ \\ ${ }^{1}$ Department of Physics and Institute for Medical \\ Engineering (IMETUM), Technische Universität München, \\ James-Franck-Strasse 1, 85748 Garching, Germany, \\ e-mail: martin.bech@tum.de \\ ${ }^{2}$ Paul Scherrer Institut, 5232 Villigen PSI, Switzerland \\ ${ }^{3}$ Niels Bohr Institut, Universitetsparken 5, 2100 \\ Copenhagen, Denmark \\ ${ }^{4}$ Lyncean Technologies Inc., 370 Portage Avenue, Palo Alto, \\ CA 94306, USA \\ ${ }^{5}$ Stanford Linear Accelerator Center, 2575 Sand Hill Road, \\ Menlo Park, CA 94025, USA \\ * Corresponding author
}

\begin{abstract}
$\mathrm{X}$-ray phase and dark-field contrast have recently been the source of much attention in the field of X-ray imaging, as they both contribute new imaging signals based on physical principles that differ from conventional X-ray imaging. With a so-called Talbot grating interferometer, both phasecontrast and dark-field images are obtained simultaneously with the conventional attenuation-based X-ray image, providing three complementary image modalities that are intrinsically registered. Whereas the physical contrast mechanisms behind attenuation and phase contrast are well understood, a formalism to describe the dark-field signal is still in progress. In this article, we report on correlative experimental results obtained with a grating interferometer and with small-angle $\mathrm{X}$-ray scattering. Furthermore, we use a proposed model to quantitatively describe the results, which could be of great importance for future clinical and biomedical applications of grating-based X-ray imaging.
\end{abstract}

Keywords: X-ray; phase contrast; dark-field; inverse Compton.

\begin{abstract}
Zusammenfassung
Die Röntgen-Bildkontrastierung mittels Phasen- und Dunkelfeld-Kontrast hat in der jüngsten Zeit besonderes Interesse erfahren, da diese Verfahren intrinsisch unterschiedliche physikalische Wechselwirkungen als Bildsignale benutzen. Mittels eines sogenannten Talbot-Interferometers werden Phasen- und Dunkelfeld-Kontrastaufnahmen gleichzeitig zusammen mit der konventionellen, abschwächungsbasierten Röntgenaufnahme erzeugt, was multimodale Röntgenbilder erlaubt, die intrinsisch registriert sind. Während die physikalischen Prozesse, die den konventionellen Röntgenaufnahmen und den Röntgen-Phasenkontrastaufnahmen zugrunde liegen gut verstanden sind, ist ein entsprechender Formalismus für die Dunkelfeldbildgebung noch in der Erforschung. In dieser Arbeit präsentieren wir experimentelle Ergebnisse, die den Zusammenhang zwischen Röntgen-Kleinwinkelstreuung und gitter-basierter Röntgen-Dunkelfeldbildgebung unter Verwendung eines laser-getriebenen Kompaktsynchrotrons unterstreichen und die für spätere klinische und biomedizinische Anwendungen von Bedeutung sein können.
\end{abstract}

Schlüsselwörter: Dunkelfeld; Laser-generierte Röntgenstrahlung; Phasenkontrast; Röntgenbildgebung; Synchrotron.

\section{Introduction}

Alternative contrast modalities in X-ray imaging, such as phase contrast and dark-field contrast, have proven to be of great value when imaging soft tissue or porous structures. In grating-based imaging, with the so-called Talbot interferometer, images with phase contrast and dark-field contrast are obtained simultaneously with the standard absorption-based X-ray image.

Although the contrast mechanism behind absorption and phase-contrast imaging is well understood, the interpretation of the dark-field image obtained with a grating interferometer is not as straight forward. Absorption- and phase-contrast images can be quantitatively described by the complex index of refraction [1-3], where attenuation and 
phase shift is described by the imaginary and real part, respectively. Dark-field contrast, on the contrary, is caused by smallangle scattering interaction in the sample, which effectively reduces the local coherence of the X-ray beam [4-6].

Attempts to make a quantitative description of the darkfield signal have been made by Wang et al. [7], Bech et al. [8], Yashiro et al. [9], Chen et al. [10], and Lynch et al. [11]. All of the proposed models suggest that the dark-field signal can be described by small-angle X-ray scattering (SAXS) or ultra-small-angle X-ray scattering (USAXS). Both Yashiro et al. [9] and Lynch et al. [11] use the approach of discerning between a smooth (resolvable) part and a fine (unresolvable) part of the wavefront.

In the work of Yashiro et al. [9], a model for the relation between the dark-field signal and the interferometer geometry (grating period and Talbot distance) is established using the autocorrelation function of the wavefront, modeled as a random Gaussian process with a Hurst exponent depending on the shape of the scattering particle. They validate the model with grating interferometer imaging experiments of microspheres and a melamine sponge performed with monochromatic synchrotron radiation. A one-dimensional SAXS scatter profile is measured at the molybdenum $\mathrm{K} \alpha$-line of an X-ray tube with silicon single-crystal monochromator and analyzer.

Lynch et al. [11] deduce a "dark-field extinction coefficient," which also depends on the autocorrelation function, and they show experimental results of monodisperse microspheres measured with a grating interferometer.

In this article, we present a quantitative study validating the Gaussian scattering distribution, which has previously been assumed [8, 12]. For each given pixel $(m, n)$ on the detector in an imaging setup, scattering in the sample introduces a Gaussian angular distribution $A_{m, n}(\theta)$ of width $\sigma_{m, n}$ :

$A_{m, n}(\theta)=\frac{1}{\sigma_{m, n} \sqrt{2 \pi}} \exp \left(-\frac{\theta^{2}}{2 \sigma_{m, n}^{2}}\right)$

where the width of the angular distribution is comparable in size to the angle spanned by a single grating period in the interferometer. The width of the beam broadening caused by the sample depends on the sample thickness, and in the case of a step-wedge of discrete thickness, the distribution can be written as a convolution corresponding to a simple addition of the Gaussian scattering width caused by each discrete step:

$\sigma_{m, n}=\sqrt{\sigma_{1, m, n}^{2}+\sigma_{2, m, n}^{2}+\cdots+\sigma_{N, m, n}^{2}}$

where $N$ is the number of steps (or sample thickness) in front of pixel $(m, n)$. To experimentally verify this assumption, we compared the dark-field image contrast from a grating interferometer operated at a monochromatic laser-based inverse Compton X-ray source $[13,14]$ with the image signal obtained from scanning SAXS microscopy [15] using monochromatic synchrotron radiation. The sample used in this study is a paper step-wedge, where the structures are too fine to be resolved by the imaging system. Paper is a good model test sample, as it contains multilength scale density variations on the relevant, i.e., few nanometers to few micrometers of length scale. The model used here is the model proposed by Bech et al. [8], which describes the dark-field signal (and hence also the small-angle scattering) as a Gaussian broadening of the beam's angular distribution.

\section{Methods}

Scanning SAXS was performed at the cSAXS beamline at the Swiss Light Source (SLS). A sketch of the setup is displayed in Figure 1A. X-rays at an energy level of 11.2 $\mathrm{keV}$ were focused to a $20-\mu \mathrm{m}$ spot size and raster-scanned across the sample. The SAXS patterns were recorded with a two-dimensional single photon counting detector (Pilatus 2M, DECTRIS Ltd., Baden, Switzerland), as described by Bunk et al. [15]. To record small scattering angles, the detector was positioned as far as possible from the sample. With a sample-to-detector distance of $7.12 \mathrm{~m}$ and a detector pixel size of $0.172 \mathrm{~mm}$, the angle covered by each pixel is $24.2 \mu \mathrm{rad}$.

Dark-field imaging with a grating interferometer was performed at the compact light source (CLS) prototype at Lyncean Technologies Inc. The CLS produces X-rays by inverse Compton scattering in a cone of $4 \mu \mathrm{rad}$ with a spectrum of $3 \%$ bandwidth [14]. In this experiment, the CLS electron storage ring was operated at a photon energy level of $21 \mathrm{keV}$, and the interferometer was placed $16 \mathrm{~m}$ from the X-ray source with
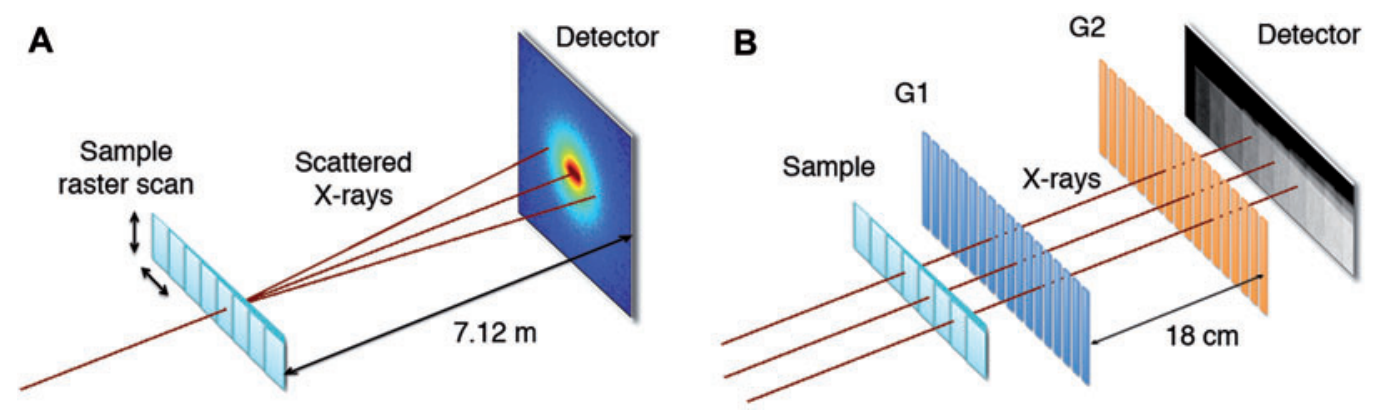

Figure 1 Sketch of experimental setup. (A) Scanning SAXS setup at the cSAXS beamline at the Swiss Light Source. The X-ray probe is raster-scanned across the sample. (B) Talbot grating interferometer at the Compact Light Source. 
a distance of $18 \mathrm{~cm}$ between the gratings corresponding to the third fractional Talbot distance [16]. The interferometer, sketched in Figure 1B, consists of two gratings: a phase grating, G1, with nickel structures, and an absorption grating, G2, with absorbing gold structures. The G1 grating had a period of $p_{1}=5.312 \mu \mathrm{m}$, with $4-\mu \mathrm{m}$-high Ni lines designed for $\pi / 2$ phase shift at $23 \mathrm{keV}$. The $\mathrm{G} 2$ grating had a period of $p_{2}=5.400 \mu \mathrm{m}$, with $55-\mu \mathrm{m}$-high $\mathrm{Au}$ lines. Both gratings were produced by microworks GmbH (Karlsruhe, Germany).

\section{Results}

To compare the dark-field image signal obtained with a grating interferometer to the scattering signal obtained with scanning SAXS, we measured a phantom made of paper with both methods. The paper phantom consisted of individual paper sheets stacked to form a step-wedge with a total thickness ranging from 0 to 10 sheets of paper, similar to the phantom described by Bech et al. [8].

Figure 2A displays the images obtained with a grating interferometer at the CLS. Figure 2B shows four synchrotron SAXS diffraction patterns of increased sample thickness. The individual frames show the direct beam and the scattering from 1, 2, 5, and 10 sheets of papers, respectively. Each frame has a width of 100 pixels, corresponding to an angular range of $2.42 \mathrm{mrad}$, or a $q$-range of $\pm 68.7 \mu \mathrm{m}^{-1}$.

Both the attenuation and dark-field images show the expected exponential behavior, as illustrated in the graph in Figure 2A, where the exponential fit is plotted together with the measured values of transmission and visibility, respectively. The exponential fits yield a linear attenuation coefficient $\mu_{1}=7.1 \times 10^{-3}$ per paper sheet and a linear diffusion coefficient [8] $\varepsilon_{1}=4.3 \times 10^{-12}$ per paper sheet.

The SAXS patterns obtained through increasing the number of paper sheets show a broadening of the beam profile, corresponding to an angular broadening of the direct beam. The four SAXS patterns displayed in Figure 2B illustrate the general broadening of the beam, and an analysis of the SAXS pattern width shows a well-behaved dependence on the number of sheets penetrated. To fit the experimental data, we assume that the direct beam has a distribution width of $\sigma_{0}$, and the angular broadening caused by one sheet of paper corresponds to a convolution with a Gaussian of width $\sigma_{1}$. Consequently the measured width after penetrating $N$ sheets of paper is expected to be

$\sigma_{\text {measured }}(N)=\sqrt{\sigma_{0}^{2}+N \sigma_{1}^{2}}$

leading to the following expression for the broadening caused by $N$ sheets of paper, $\sigma_{N}$ :

$\sigma_{N}=\sigma_{1} \sqrt{N}=\sqrt{\sigma_{\text {measured }}^{2}(N)-\sigma_{0}^{2}}$

A plot of the beam broadening caused by the paper stepwedge is displayed in Figure 3. The measured broadening $\sigma_{N}$ caused by $N$ sheets of paper is plotted as blue circles together with a red line indicating the expected behavior according to the model Eqn. (4). In the calculation of $\sigma_{0}$, a slightly modified Gauss profile has been used to correct for the fact that a Gaussian angular distribution of the direct beam is not experimentally achievable, as the slits used to shape the beam can only create binary-like beam distributions.

\section{Discussion and conclusion}

All models describing dark-field X-ray imaging contain Gaussian scattering distributions. The SAXS results presented here show that the approximation of Gaussian scattering distribution is valid for optically thin samples, in this particular case, paper stacks of step-like thickness. In this study, we have shown that a Gaussian distribution is valid for investigating multiscale density variations below the resolution limit. The measurements of a paper test sample containing fine (unresolvable) microstructures follow the proposed model well, as measured directly with a state-of-the-art SAXS setup at the SLS: the width of the angular broadening of the direct beam caused by scattering in the sample is proportional to the square root of the sample thickness.
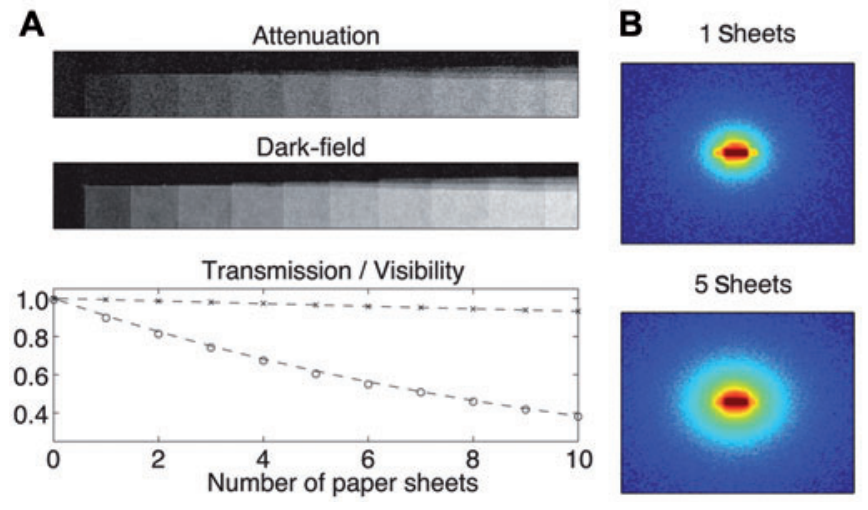

5 Sheets

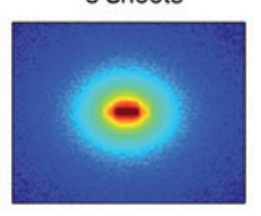

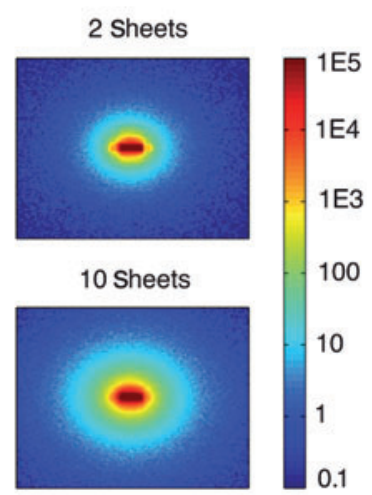

Figure 2 (A) Attenuation and dark-field images and profiles of paper step-wedge measured with a Talbot grating interferometer at a laserdriven compact X-ray source (crosses=transmission through sample; dots=visibility through sample). (B) SAXS patterns from paper stepwedge measured with monochromatic synchrotron radiation. The width of each pattern corresponds to a $q$ range of $\pm 68.7 \mu \mathrm{m}^{-1}$. 


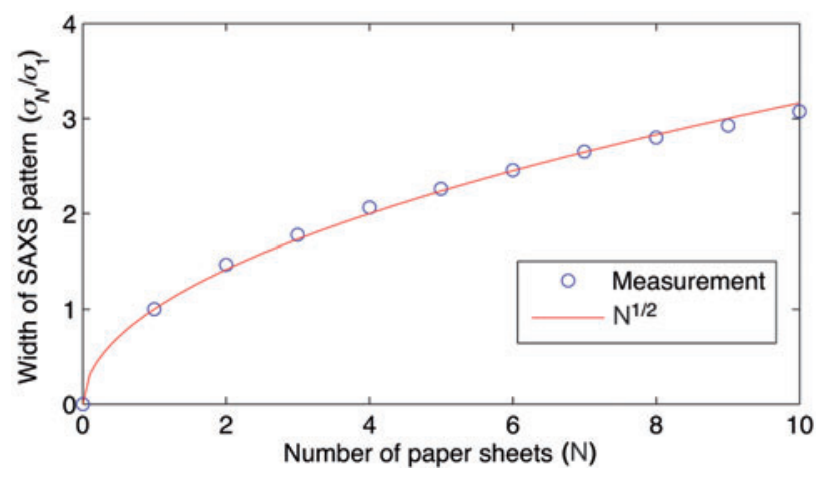

Figure 3 Width of Gaussian broadening caused by paper stepwedge. The measured values $\sigma_{\text {measured }}$ have been corrected by the width of the direct beam $\sigma_{0}$ as indicated in Eq. (4) and normalized to $\sigma_{1}$.

We consider dark-field imaging to be of great importance for future clinical and biomedical applications of gratingbased X-ray imaging. The large field of view allowed by the grating interferometer makes clinical imaging possible, and with the quasi-monochromatic X-ray beam provided by the inverse Compton-based CLS, high-quality dark-field and phase-contrast images can be envisioned in the near future. The results could contribute to improving the quality of X-ray imaging, thus extending the field of application for the investigation of, for example, laser-induced damage to the cytoskeleton and for medical laboratory diagnostics.

\section{Acknowledgments}

M.B., S.S., G.P., K.A., and F.P. acknowledge financial support from the DFG Cluster of Excellence Munich - Center for Advanced Photonics (DFG EXC-158) and the European Research Council (FP7, Starting Grant 24012). The CLS was partially supported by the NIH through grants R44-GM074437 and U54-GM074961 from NIGMS and NCRR and R43-RR025730 from NCRR.

\section{Conflict of interest statement}

Professor Ronald Ruth's contributions to this publication were as a paid consultant of Lyncean Technologies Inc. and were not part of his Stanford University duties or responsibilities.

\section{References}

[1] Als-Nielsen J, McMorrow D. Elements of modern X-ray physics. Chichester: John Wiley \& Sons; 2001.
[2] Momose A. Recent advances in X-ray phase imaging. Jpn J Appl Phys 2005;44:6355-67.

[3] Weitkamp T, Diaz A, David C, Pfeiffer F, Stampanoni M, Cloetens P, Ziegler E. X-ray phase imaging with a grating interferometer. Opt Express 2005;13(16):6296-304.

[4] Pfeiffer F, Bech M, Bunk O, Kraft P, Eikenberry EF, Brönnimann Ch, Grünzweig C, David C. Hard-X-ray dark-field imaging using a grating interferometer. Nat Mater 2008;7(2):134-7.

[5] Wen H, Bennett EE, Hegedus MM, Carroll SC. Spatial harmonic imaging of X-ray scattering -initial results. IEEE Trans Med Imaging 2008;27(8):997-1002.

[6] Jensen TH, Bech M, Zanette I, Weitkamp T, David C, Deyhle H, Rutishauser S, Resnikova E, Mohr J, Feidenhans'1 R, Pfeiffer F. Directional X-ray dark-field imaging of strongly ordered systems. Phys Rev B 2010;82:214103.

[7] Wang ZT, Kang KJ, Huang ZF, Chen ZQ. Quantitative gratingbased X-ray dark-field computed tomography. Appl Phys Lett 2009;95(9):094105.

[8] Bech M, Bunk O, Donath T, Feidenhans'l R, David C, Pfeiffer F. Quantitative X-ray dark-field computed tomography. Phys Med Biol 2010;55(18):5529-39.

[9] Yashiro W, Terui Y, Kawabata K, Momose A. On the origin of visibility contrast in X-ray Talbot interferometry. Opt Express 2010;18(16):16890-901.

[10] Chen GH, Bevins N, Zambelli J, Qi Z. Small-angle scattering computed tomography (SAS-CT) using a Talbot-Lau interferometer and a rotating anode X-ray tube: theory and experiments. Opt Express 2010;18(12):12960-70.

[11] Lynch SK, Pai V, Auxier J, Stein AF, Bennett EE, Kemble CK, Xiao X, Lee WK, Morgan NY, Wen HH. Interpretation of darkfield contrast and particle-size selectivity in grating interferometers. Appl Opt 2011;50(22):4310-9.

[12] Bech M, Jensen TH, Bunk O, Donath T, David C, Weitkamp T, Le Duc G, Bravin A, Cloetens P, Pfeiffer F. Advanced contrast modalities for X-ray radiology: phase-contrast and darkfield imaging using a grating interferometer. Z Med Phys 2010;20(1):7-16.

[13] Huang Z, Ruth RD. Laser-electron storage ring. Phys Rev Lett 1998;80:976-9.

[14] Bech M, Bunk O, David C, Ruth R, Rifkin J, Loewen R, Feidenhans'l R, Pfeiffer F. Hard X-ray phase-contrast imaging with the Compact Light Source based on inverse Compton X-rays. J Synchrotron Radiat 2009;16(Pt 1):43-7.

[15] Bunk O, Bech M, Jensen TH, Feidenhans'l R, Binderup T, Menzel A, Pfeiffer F. Multimodal X-ray scatter imaging. New J Phys 2009; 11:123016.

[16] Weitkamp T, Zanette I, David C, Baruchel J, Bech M, Bernard P, Deyhle H, Donath T, Kenntner J, Lang S, Mohr J, Müller B, Pfeiffer F, Reznikova E, Rutishauser S, Schulz G, Tapfer A, Valade J-P. Recent developments in X-ray Talbot interferometry at ESRF-ID19. Proc SPIE 2010;7804:780406.

Received November 4, 2011; revised December 5, 2011; accepted December 8, 2011 